\title{
NEW KINK-LIKE SOLUTIONS FOR NONLINEAR EQUATION DESCRIBING THE DYNAMICS OF DNA
}

\author{
M. A. Knyazev ${ }^{1}$, D. M. Knyazev ${ }^{2}$ \\ ${ }^{1}$ Chair of Technical Physics, Belarussian National Technical University, \\ Pr. Nezalezhnasti, 65, Minsk 220013, Belarus, \\ ${ }^{2}$ EPAM-Systems, Academician Kuprevich Street, 1/1, Minsk 220141, Belarus
}

(Received May 17, 2011)

\begin{abstract}
The nonlinear model of DNA is considered. In this model only the longitudinal and transverse modes are taken into account but the helical structure and torsional motion are neglected. The new kink-like solutions for the nonlinear equation of motion which describes the stretch of the hydrogen bond are constructed by means of the Hirota method.
\end{abstract}

Key words: kink, Hirota method, Deoxyribonucleic acid, nonlinear dynamics.

PACS number(s): 02.30.Jr, 03.40.Kf, 87.14.Cg

\section{INTRODUCTION}

Deoxyribonucleic acid (DNA) molecule consists of two long elastic rods with a helical structure. This molecule is a carrier of information for the life and reproduction of organisms. Because of this it used to be and continues to be a very important subject of experimental and theoretical studies. The kink and kink-like states are widely used for the description the nonlinear dynamics of the DNA molecule. In [1] the steady-state torsional stress and shape of a rotating rod with a kink was calculated. The impact of the kinks on the deformation and torsional stress of a twirling elastic rod is demonstrated. The effects of the genetic sequence on the propagation of nonlinear excitations in some simple models of DNA was studied in [2]. The known result that kink propagation requires forces over a certain threshold was confirmed. Below the threshold the dependence of the kink dynamics on the information content of the genetic sequences was not observed. A worm-like polymer chain with reversible kink-like structural defects was considered in [3]. This model is used for the description of a generic model for the double-stranded DNA with sharp bends induced by binding of certain proteins and the effects of trans-gauche rotations in the backbone of the single-stranded DNA. It was found that the persistence length is renormalized due to the presence of the kinks. In [4] an existence of stable kink (antikink) states in a model of DNA which account the dissipative effect of intermolecular vibrational modes was found. An investigation of the probability that a portion of DNA closes on itself through thermal fluctuations as a function of the presence of a kink at half DNA length is presented in [5]. A kink and antikink states are used in [6] for a study of motion of semiflexible biopolymers as DNA in double-well potentials. It was shown that the activated dynamics of such polymers is governed by the nucleation of localized kink-like excitations. For the nonlinear dynamic plane-base rotator model of an inhomogeneous DNA double helical chain the variations of perturbations on the formation of kink-antikink states were considered in [7]. In paper [8] it was shown how the formation of a single permanent kink changes the DNA force-extension relation in the finite worm-like chain model of short DNA molecules attached to beads. In [9] on the base of a combined model which includes two well-known models (the pendulum model of Englander [10] and the microscopic model of Peyrard and Bishop [11]) the nonlinear excitations in a DNA molecule were studied as a function of temperature. It was demonstrated that the kink velocity depends on temperature. The formation of the kinks in a cyclized DNA molecule when an anisotropy is taken into account was considered in [12]. A model for DNA dynamics by introducing the helical structure through twist deformation by analogy with the structure of helimagnet and cholesteric liquid crystal system was proposed in [13]. In this model the sine-Gordon equation is used which admits kink-antikink solutions. The helicity leads to a length scale variation that provides a better representation of the base pair opening in DNA. The second variation of DNA rings is investigated in [14]. On the base of the Kirchhoff elastic theory a stability analysis of kinked DNA is realized. Taking into account the anharmonic corrections to the anisotropic elastic rod model for DNA allowed to explain a kink formation at high deformation limit [15].

A further study of such nonlinear localized excitations is of great interest. A construction of new kink- or kinklike solutions will allow to describe some new peculiarities of the dynamics for DNA molecules and explain the mechanisms of their behavior.

In this paper the new one kink-like solutions for the model of a DNA molecule in which the longitudinal and transverse modes are taken into account are studied. In Section II a model of DNA is described and the nonlinear equation of motion is considered. In Section III the solutions of this equation are constructed by the Hirota method and the conditions on the parameters of solutions additionally to the conditions on the parameters of the model are pointed out. In Conclusion some final remarks are contained. 


\section{MODEL AND EQUATION}

Let us consider a continuous model for a DNA molecule developed in [16] which is based on the lattice model presented in [11]. We will follow [17] in the description of the model. The two long elastic rods of a DNA molecule represent two polynucleotide chains. They are connected by an elastic membrane. This membrane represents the hydrogen bonds between the pairs of bases in the chains. As a DNA molecule is supposed to be homogeneous both rods have the same mass density. The helical structure of DNA is neglected. The only longitudinal and transverse motions of DNA rods are considered.
The torsion motion is neglected. The model has four degrees of freedom: $u_{1}$ and $u_{2}$ represent respectively, the longitudinal displacements of the top and bottom rods (displacements of the bases from equilibrium positions along the direction of the phosphodiester bridge between the two bases of the same rod); $\nu_{1}$ and $\nu_{2}$ represent respectively the transverse displacements of the top and bottom rods (displacements of the bases from equilibrium positions along the direction of the hydrogen bonds).

The Hamiltonian has a form

$$
H=T+V_{1}+V_{2},
$$

where the kinetic energy of elastic rods

$$
T=\int \frac{1}{2} \rho \sigma\left[\left(\frac{\partial u_{1}}{\partial t}\right)^{2}+\left(\frac{\partial u_{2}}{\partial t}\right)^{2}+\left(\frac{\partial \nu_{1}}{\partial t}\right)^{2}+\left(\frac{\partial \nu_{2}}{\partial t}\right)^{2}\right] d x
$$

the potential energy of elastic rods

$$
V_{1}=\int \frac{1}{2} Y \sigma\left[\left(\frac{\partial u_{1}}{\partial x}\right)^{2}+\left(\frac{\partial u_{2}}{\partial x}\right)^{2}\right] d x+\int \frac{1}{2} F \sigma\left[\left(\frac{\partial \nu_{1}}{\partial x}\right)^{2}+\left(\frac{\partial \nu_{2}}{\partial x}\right)^{2}\right] d x
$$

and the potential energy of elastic membrane

$$
V_{2}=\int \frac{1}{2} \mu[\Delta l(x)]^{2} d x
$$

Here $\rho, \sigma Y$ and $F$ are the mass density, the area of transverse cross-section, the Young's modulus and the tension density of each rod; $\mu$ is the rigidity of the elastic membrane; the stretching of the elastic membrane at $x$ due to longitudinal vibrations

$$
\Delta l=\sqrt{\left(h+\nu_{1}-\nu_{2}\right)^{2}+\left(u_{2}-u_{1}\right)^{2}}-l_{0},
$$

$h$ is the distance between the two rods, $l_{0}$ is the height of the membrane in the equilibrium.

To obtain the equation of motion let us introduce the new variables: for in-phase motion

$$
u_{+}=\frac{u_{1}+u_{2}}{\sqrt{2}} \quad \text { and } \quad \nu_{+}=\frac{\nu_{1}+\nu_{2}}{\sqrt{2}} ;
$$

for out-phase motion

$$
u_{-}=\frac{u_{2}-u_{1}}{\sqrt{2}} \quad \text { and } \quad \nu_{-}=\frac{\nu_{2}-\nu_{1}}{\sqrt{2}} .
$$

Then assume that

$$
\left|u_{1}-u_{2}\right| \ll h, \quad\left|\nu_{1}-\nu_{2}\right| \ll h .
$$

It allows to use the expansion

$$
\begin{aligned}
\frac{\Delta l}{l_{0}+\Delta l} & =1-\frac{l_{0}}{h}+\frac{l_{0}}{h^{2}}\left(\nu_{1}-\nu_{2}\right)-\frac{l_{0}}{h^{3}}\left(\nu_{1}-\nu_{2}\right)^{2} \\
& +\frac{l_{0}}{2 h^{3}}\left(u_{2}-u_{1}\right)^{2}
\end{aligned}
$$

and drop the higher order terms. The out-of-phase motion stretches the hydrogen bond. That is why only this motion will be considered. By introducing the transformation $\nu_{-}=a u_{-}+b$, where $a$ and $b$ are some constants and putting $u_{-}=\phi$, the equation of motion may be written in the form

$$
\phi_{t t}-c_{1}^{2} \phi_{x x}=A \phi^{3}+B \phi^{2}+C \phi
$$

with

$$
\phi_{t t}=\frac{\partial^{2} \phi}{\partial t^{2}} \quad \text { and } \quad \phi_{x x}=\frac{\partial^{2} \phi}{\partial x^{2}}
$$

Here

$$
\begin{gathered}
c_{1}^{2}=\frac{Y}{\rho}, \quad A=\left(\frac{-2 \alpha}{h^{3}}+\frac{4 a^{2} \alpha}{h^{3}}\right), \quad B=\frac{6 \sqrt{2} a \alpha}{h^{2}}, \\
C=\left(\frac{-2 \alpha}{l_{0}}+\frac{6 \alpha}{h}\right), \quad \alpha=\frac{\mu l_{0}}{\rho \sigma} .
\end{gathered}
$$

For $b$ one has $b=\frac{h}{\sqrt{2}}$; as regards the parameter $a$, it is still arbitrary. 
Eq. (2) has the exact kink-like solution

$$
\phi=\frac{-\sqrt{2} a h}{2 a^{2}-1}\left[1 \pm \tanh \left(\sqrt{\frac{2 a^{2} \mu l_{0}}{\rho \sigma h\left(2 a^{2}-1\right)}} \xi\right)\right]
$$

that may be obtained by using the elliptic equation. Here $\xi=\left(x-\sqrt{2} c_{1} t\right) / c_{1}, a^{2}>1 / 2$ and plus(minus) corresponds for antikink(kink), respectively. In the next section the new solutions of such a type will be obtained by the Hirota method.

\section{KINK-LIKE SOLUTIONS}

To solve Eq. (2) let us introduce a new dependent variable by the Cole-Hopf transformation

$$
\phi=\sigma \frac{F_{x}}{F},
$$

where $\sigma$ is a parameter to be determined and $F=F(x, t)$ is a new unknown function. Substituting Eq. (4) into Eq. (2) results in the following nonlinear partial differential equation

$$
\begin{aligned}
F_{x t t} F^{2} & -2 F_{x t} F_{t} F-F_{x} F_{t t} F+2 F_{x} F_{t}^{2}-c_{1}^{2} F_{x x x} F^{2} \\
& -3 c_{1}^{2} F_{x} F_{x x} F-C F_{x} F^{2}-\sigma B F_{x}^{2} F=0 .
\end{aligned}
$$

This equation is obtained under the condition

$$
2 c_{1}^{2}+A \sigma^{2}=0
$$

which may be fulfilled for $a^{2}<1 / 2$. From this condition a the value of $\sigma$ is determined.

Now we represent the function $F$ as a formal series

$$
F=1+\varepsilon f_{1}+\varepsilon^{2} f_{2}+\ldots,
$$

where $f_{i}(x, t), i=1,2, \ldots$ are the new unknown functions and $\varepsilon$ is not, generally speaking, a small constant. By substituting Eq. (6) into Eq. (5) and equating to the zero coefficients for each degree of $\varepsilon$, one obtains the infinite system of linear partial differential equations for functions $f_{i}$. The two first equations of this system should be used. They have the form

$$
\begin{gathered}
f_{1, x t t}-c_{1}^{2} f_{1, x x x}-C f_{1, x}=0 \\
f_{2, x t t}-c_{1}^{2} f_{2, x x x}-C f_{2, x}=2 f_{1, x t} f_{1, t}+f_{1, x} f_{1, t t} \\
-3 c_{1}^{2} f_{1, x} f_{1, x x}+\sigma B f_{1, x}^{2} .
\end{gathered}
$$

To construct one-kink solution one needs the function $f_{1}$ only. Let us represent the function $f_{1}$ in the form

$$
f_{1}(x, t)=\exp \left(k x-\omega t+\eta^{0}\right),
$$

where $k, \omega$ and $\eta^{0}$ are some parameters. The parameter $\eta^{0}$ corresponds to the initial phase and may be putting to zero. To determine the parameters $k$ and $\omega$ two equations are needed. By substituting Eq. (9) into Eq. (7) one obtains the first relation between $k$ and $\omega$

$$
\omega^{2}=c_{1}^{2} k^{2}+C
$$

The second linearly independent equation to determine $k$ and $\omega$ is obtained from the requirement of breaking the series (6) in the case of one-kink solution. It means that the right-hand side of Eq. (8) should be equal to zero. This leads to the equation

$$
3 \omega^{2}-3 c_{1}^{2} k^{2}+\sigma B k=0 .
$$

By solving Eqs.(10) and (11) one obtains

$$
k=-\frac{3 C}{\sigma B} \quad \text { and } \quad \omega^{2}=\frac{9 C^{2} c_{1}^{2}+\sigma^{2} B^{2} C}{\sigma^{2} B^{2}} .
$$

Finally, new one-kink solutions take the form

$$
\phi=\sigma \frac{f_{1, x}}{f_{1}}=\frac{\sigma k}{2}\left[1+\tanh \left(\frac{k x-\omega t}{2}\right)\right] .
$$

The only parameter $a$ is arbitrary. All the other parameters in Eq. (13) are determined. Depending on their signs this equation gives both kink-like and antikink-like solutions. The condition $\omega^{2}>0$ results in the next relation between the parameters

$$
3 \alpha\left(2 a^{2}-1\right)\left(3 l_{0}-h\right)>\sqrt{2} l_{0} h a
$$

For the case $a^{2}<1 / 2$ this equation leads to the condition $h>3 l_{0}$. This is in agreement with the physical conditions on the parameters of the model.

\section{CONCLUSION}

The nonlinear model for DNA is studied. The DNA molecule is considered as consisting of two long elastic rods which are connected by the elastic membrane. In this model the longitudinal and transverse modes are taken into account only. The helical structure and torsional motion are neglected. The equation of motion is considered for the so-called out-of-phase motion which stretches the hydrogen bond. The new one kink-like and antikink-like solutions for this equation are constructed by means of the Hirota method. One can conclude that each of the obtained solutions is stable because its velocity is a constant. One can note that for these solutions the Riccati generalization suggested in [17] is possible. 
[1] S. A. Koehler, T. R. Powers, Phys. Rev. Lett. 85, 4827 (2000).

[2] S. Cuenda, A. Sanchez, preprint arXiv:q-bio/0407002 (2004).

[3] Yu. O. Popov, A. Tkachenko, Phys. Rev. E 71, 051905 (2005).

[4] N. L. Komarova, A. Soffer, preprint arXiv:cond-mat/ 0411621 (2004).

[5] N. Douarche, S. Cocco, Phys. Rev. E 72, 061902 (2005).

[6] P. Kraikivski, R. Lipowsky, J. Kierfeld, Europhys. Lett. 66, 763 (2004).

[7] M. Daniel, V. Vasumathi, Physica D 231, 10 (2007).

[8] Jinyu Li, P. C. Nelson, M. D. Betterton, Macromolecules 39, 8816 (2006).

[9] D. L. Hien, N. T. Nhan, V. Thanh Ngo, N. A. Viet, Phys. Rev. E 76, 021921 (2007).
[10] S. W. Englander, N. R. Kallenback, A. J. Heeger, J. A. Krumhanst, S. Kitwin, Proc. Natl. Acad. Sci. USA 77, 7222 (1980).

[11] M. Peyrard, A. R. Bishop, Phys. Rev. Lett. 62, 2755 (1989).

[12] D. Norouzi, F. Mohammad-Rafiee, R. Golestanian, Phys. Rev. Lett. 101, 168103 (2008).

[13] M. Daniel, V. Vasumathi, preprint arXiv:0812.4536 (2008).

[14] Xiao-hua Zhou, preprint arXiv:0901.0456 (2009).

[15] B. Eslami-Mossallam, M. R. Ejtehad, preprint arXiv: 0904.4008 (2009).

[16] D. X. Kong, S. Y. Lou, J. Zeng, Commun. Theor. Phys. 36, 737 (2001).

[17] W. Alka, A. Goyal, C. Nagaraja Kumar, Phys. Lett. A 375, 480 (2011).

\title{
НОВІ ПЕТЛЕПОДІБНІ РОЗВ'ЯЗКИ НЕЛІНІЙНОГО РІВНЯННЯ, ЩО ОПИСУЕ ДИНАМІКУ ДНК
}

\author{
М. А. Князєв ${ }^{1}$, Д. М. Князєв ${ }^{2}$ \\ ${ }^{1}$ Кафедра технічної фізики, Білорусъкий начіональний технічний університет, \\ Мінсък, 220013, Білорусъ, \\ 2 EPAM-Systems, Мінсък, 220141, Білорусъ
}

Розглянуто нелінійну модель ДНК. У ній враховують лише поздовжні та поперечні моди, а спіральною структурою й обертовими рухами нехтують. За допомогою методу Гіроти сконструйовано нові петлеподібні розв'язки нелінійного рівняння руху, яке описує розтягування водневого зв'язку. 